\title{
Propuesta del Plan de Manejo Ambiental de las Lagunas de Mojanda: Caricocha, Huarmicocha y Yanacocha del cantón Pedro Moncayo
}

Proposal for the environmental management plan for the lagunas de Mojanda: Caricocha, Huarmicocha and Yanacocha of the Pedro Moncayo Canton

Proposta de plano de gestão ambiental das lagoas de Mojanda: Caricocha, Huarmicocha e Yanacocha do cantão Pedro Moncayo

Paul N. Malacatus-Cobos I

pnmalacatus@uce.edu.ec

Byron V. Guerrero-Rodríguez II

bvguerreror@uce.edu.ec

Gerardo M. Llerena-Carrera ${ }^{\text {III }}$

gmllerenac@uce.edu.ec

Recibido: 30 de enero de 2017 * Corregido: 20 de febrero de 2017 * Aceptado: 20 mayo de 2017

I. Magister en Sistemas de Gestión; Ingeniero en Gestión Ambiental; Docente Ingeniería Ambiental; Universidad Central del Ecuador, Quito, Ecuador.

II. Master of Science in Leadership; Diploma Superior en Docencia Universitaria; Ingeniero Electrónico en Control y Automatización; Docente Ingeniería en Minas; Universidad Central del Ecuador, Quito, Ecuador.

III. Magister en Administración de Empresas (MBA) Programa Integral de Habilidades Múltiples; Ingeniero en Geología; Docente Ingeniería en Minas; Universidad Central del Ecuador, Quito, Ecuador. 


\section{Resumen}

La Universidad Central del Ecuador a través de la Carrera de Ingeniería Ambiental de la Facultad de Ingeniería en Geología, Minas, Petróleos y Ambiental; desde abril del 2016 se integra al proyecto de vinculación con la sociedad del GAD Pedro Moncayo conjuntamente con la participación de otras facultades de la institución.

Los estudiantes de Ingeniería Ambiental de noveno semestre promoción abril-agosto 2016, bajo la supervisión, de los Autores,inicianel proyecto el día 18 de abril del 2016 con una previa capacitación y una posterior visita de campo el día 20 de abril del 2016, a las lagunas de Mojanda: Caricocha, Huarmicocha y Yanacocha, ubicadas en el cantón Pedro Moncayo, donde se realiza un primer diagnóstico, en el que se identifica la pérdida de ecosistemas nativos, debido a la falta de control de las actividades turísticas que se desarrollan en esta zona.

Es así que se plantea la elaboración de una propuesta del Plan de Manejo Ambiental de las Lagunas de Mojanda: Caricocha, Huarmicocha y Yanacocha; con el fin de estableceracciones para prevenir, mitigar, controlar, compensar y corregir los posibles efectos o impactos ambientales negativos causados por las actividades desarrolladas en dicha zona.

Palabras clave: Plan; manejo; ambiental. 


\section{Abstract}

The Central University of Ecuador through the Environmental Engineering Career of the Faculty of Engineering in Geology, Mines, Petroleum and Environmental; since April 2016 he joined the project linking with the society of the GAD Pedro Moncayo jointly with the participation of other faculties of the institution.

The students of Environmental Engineering of the ninth semester of the April-August 2016 promotion, under the supervision of the authors, started the project on April 18, 2016 with prior training and a subsequent field visit on the 20th. April 2016, to the lagoons of Mojanda: Caricocha, Huarmicocha and Yanacocha, located in the canton of Pedro Moncayo, where a first diagnosis is made, in which the loss of native ecosystems is identified, due to the lack of control of the activities tourist activities that take place in this area.

This is how the proposal of the Environmental Management Plan for the Lagunas de Mojanda: Caricocha, Huarmicocha and Yanacocha is proposed; in order to establish actions to prevent, mitigate, control, compensate and correct the possible negative environmental effects or impacts caused by the activities carried out in said area.

Key words: Plan; management; environmental. 


\section{Introducción.}

El intercambio de experiencias entre la Universidad Central del Ecuador, las comunidades del cantón Pedro Moncayo y el Gobierno Autónomo Descentralizado, constituye una oportunidad para desarrollar el potencial de los estudiantes, y entrar en procesos de reflexión, de valoración, de crítica constructiva de todo aquello que ocurre a su alrededor, propiciando una ocasión valiosa para dialogar, compartir, integrarse y fortalecer el proceso de socialización y vinculación de la institución educativa con la comunidad.

En estas consideraciones se propone el presente proyecto que permitirá a los docentes y estudiantes de la Carrera de Ingeniería Ambiental de la Universidad Central del Ecuador formular soluciones mediante la propuesta de acciones técnicas, con la aplicación de los conocimientos adquiridos en el proceso de su formación profesional.

El proyecto es factible de realización ya que cuenta con el talento humano como son los docentes, estudiantes, líderes comunitarios y técnicos del GAD Pedro Moncayo; y no requiere de gran cantidad de recursos económicos para su ejecución, basta con el compromiso de los involucrados en el proyecto.

\section{Material y métodos.}

Se empleó la Metodología de Marco Lógico, se realizó en primer lugar una Matriz de involucrados para conocer e identificar las necesidades de los interesados del proyecto. 
Propuesta del Plan de Manejo Ambiental de las Lagunas de Mojanda: Caricocha, Huarmicocha y Yanacocha del cantón Pedro Moncayo

\begin{tabular}{|l|l|l|l|}
\hline Grupos & Problemas percibidos & Recursos y Mandatos & Interés en el proyecto \\
\hline & & & \\
\hline
\end{tabular}

\section{Tabla 1. Estructura de Matriz de involucrados}

Así también se estructuró un árbol de problemas que permitió desglosar el problema, las causas y los efectos de la falta de una Propuesta de Plan de Manejo de las Lagunas en Mojanda.

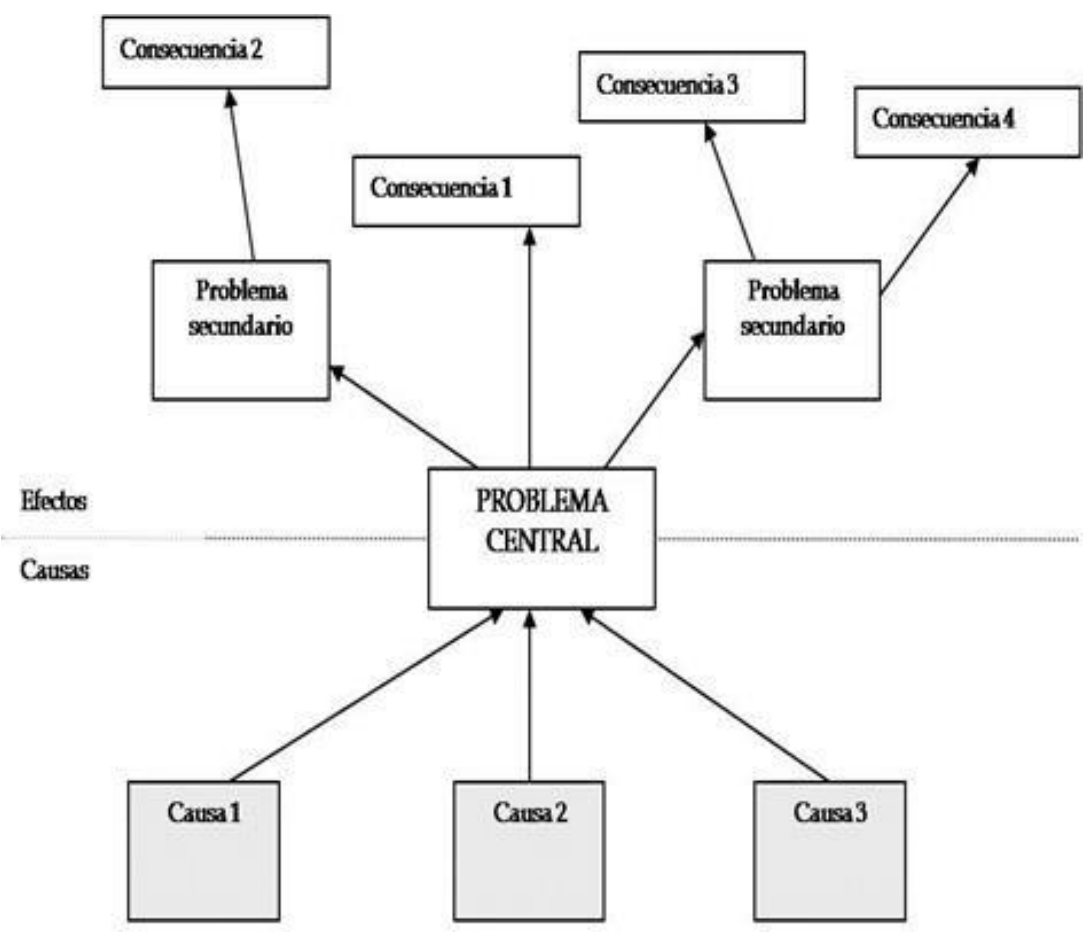

Figura 1. Estructura de árbol de problemas

Se estableció un árbol de objetivos para definir los criterios de evaluación de las distintas soluciones del problema. 


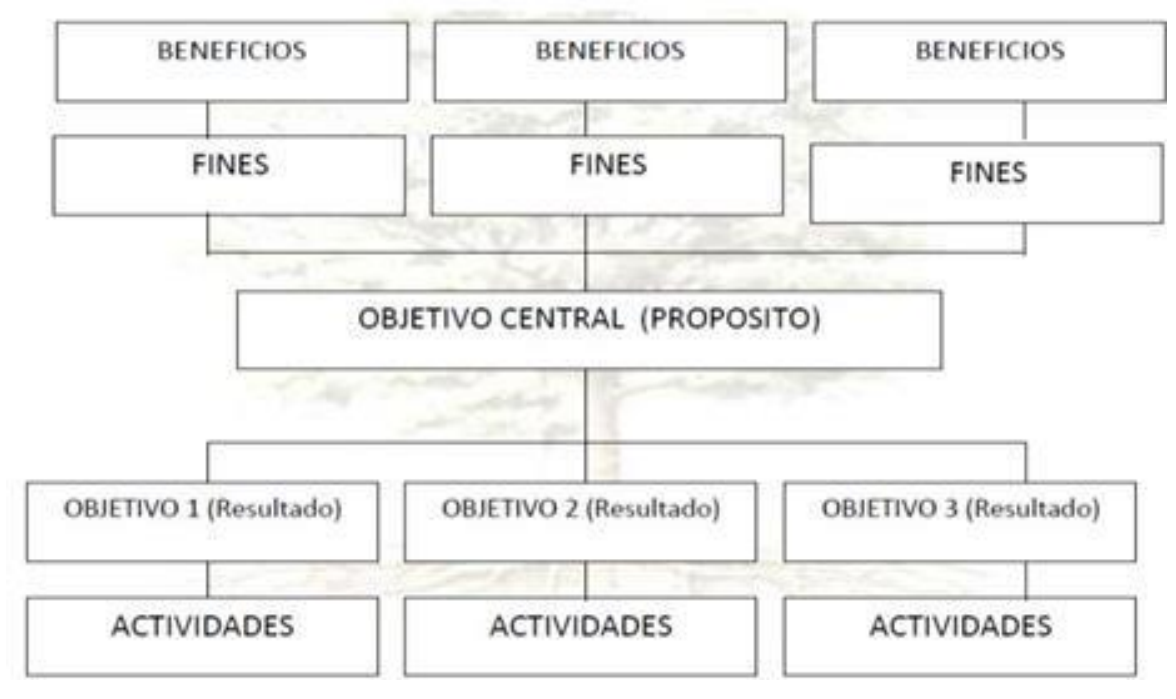

Figura 2. Estructura de árbol de objetivos

Se elaboró la Matriz de Marco Lógico que permitió realizar la planificación del proyecto mediante la presentación de forma sistemática y lógica los objetivos y sus relaciones de causalidad y las diferentes actividades a desarrollarse.

\begin{tabular}{|c|c|c|c|}
\hline DESCRIPCIÓN & \multirow{2}{*}{ INDICADORES } & $\begin{array}{c}\text { FUENTE DE } \\
\text { VERIFICACIÓN }\end{array}$ & SUPUESTOS \\
\cline { 1 - 1 } FIN & & & \\
\cline { 1 - 1 } & & & \\
\cline { 1 - 1 } PROPÓSITO & & & \\
\cline { 1 - 1 } COMPONENTES & & & \\
\cline { 1 - 1 } & & & \\
\cline { 1 - 1 } ACTIVIDADES & & & \\
\cline { 1 - 1 } & & & \\
\hline
\end{tabular}

Tabla 2. Estructura de Matriz de Marco Lógico 
Dom. Cien., ISSN: 2477-8818

Vol. 3, núm. 3, mon., agosto, 2017, pp. 811-828

Propuesta del Plan de Manejo Ambiental de las Lagunas de Mojanda: Caricocha, Huarmicocha y Yanacocha del cantón Pedro Moncayo

Se elaboró una Matriz FODA, con el fin de determinar las fortalezas, oportunidades

debilidades y amenazas del proyecto en 4 aspectos: sociales, económicos, ambientales y legislativos.

\begin{tabular}{|c|c|}
\hline \multicolumn{2}{|c|}{ FORTALEZAS } \\
\hline Sociales & \\
\hline Económicas & \\
\hline Ambientales & \\
\hline Legislativas & DEBILIDADES \\
\hline Sociales & \\
\hline Económicas & \\
\hline Ambientales & \\
\hline Legislativas & \\
\hline & OPORTUNIDADES \\
\hline Sociales & \\
\hline Económicas & \\
\hline Ambientales & \\
\hline Legislativas & \\
\hline \multicolumn{2}{|c|}{} \\
\hline Sociales & AMENAZAS \\
\hline Económicas & \\
\hline Ambientales & \\
\hline Legislativas & \\
\hline
\end{tabular}

Tabla 3. Estructura de Matriz FODA

Se estructuró la Propuesta de Plan de Manejo Ambiental, constituido por los siguientes programas enunciados a continuación siguiendo el formato descrito en la Tabla 3:

1. Programa de administración, control y vigilancia 
Dom. Cien., ISSN: 2477-8818

Vol. 3, núm. 3, mon., agosto, 2017, pp. 811-828

Propuesta del Plan de Manejo Ambiental de las Lagunas de Mojanda: Caricocha, Huarmicocha y Yanacocha del cantón Pedro Moncayo

2. $\quad$ Programa de planificación participativa

3. Programa de desarrollo comunitario y educación ambiental

4. Programa de turismo y recreación

5. Programa de investigación, manejo de recursos y monitoreo

6. Programa de sostenibilidad financiera

\begin{tabular}{|c|c|c|c|c|c|}
\hline \multicolumn{6}{|c|}{ PROGRAMA DE } \\
\hline \multicolumn{5}{|c|}{ OBJETIVO: } & \multirow{3}{*}{ PSF-... } \\
\hline \multicolumn{5}{|c|}{ LUGAR DE APLICACIÓN: } & \\
\hline \multicolumn{5}{|c|}{ RESPONSABLE(S): } & \\
\hline $\begin{array}{l}\text { OBJETIVOS } \\
\text { ESPECÍFICOS }\end{array}$ & $\begin{array}{l}\text { LÍNEAS DE } \\
\text { ACCIÓN }\end{array}$ & ACTIVIDADES & INDICADORES & $\begin{array}{l}\text { MEDIOS DE } \\
\text { VERIFICACIÓN }\end{array}$ & PLAZO \\
\hline & & & & & \\
\hline
\end{tabular}

Tabla 4. Estructura de los programas de la Propuesta del Plan de Manejo Ambiental

\section{Resultados.}

Al utilizar la metodología de Marco Lógico, se obtuvo como resultados Matriz de involucrados del proyecto:

\begin{tabular}{|c|c|c|c|}
\hline Grupos & Problemas percibidos & Recursos y Mandatos & Interés en el proyecto \\
\hline GAD Pedro Moncayo & $\begin{array}{c}\text { No puede conseguir la } \\
\text { declaración de área } \\
\text { protegida de la zona de } \\
\text { estudio. }\end{array}$ & $\begin{array}{c}\text { Obtención de declaración } \\
\text { de área protegida. }\end{array}$ & $\begin{array}{c}\text { Conservar los ecosistemas } \\
\text { propios de la zona. }\end{array}$ \\
\hline $\begin{array}{c}\text { Dueños de los predios } \\
\text { de las lagunas }\end{array}$ & $\begin{array}{c}\text { No aceptan indemnizaciones } \\
\text { por los predios ubicados en } \\
\text { la zona de estudio. }\end{array}$ & $\begin{array}{c}\text { Llegar a un acuerdo mutuo } \\
\text { entre el GAD y los } \\
\text { propietarios. }\end{array}$ & $\begin{array}{c}\text { Conservar sus propiedades u } \\
\text { obtener indemnizaciones. }\end{array}$ \\
\hline $\begin{array}{c}\text { Universidad Central } \\
\text { (Departamento de } \\
\text { Vinculación con la }\end{array}$ & Falta de organización. & $\begin{array}{c}\text { Otorgar los recursos } \\
\text { necesarios para la }\end{array}$ & $\begin{array}{c}\text { Conseguir convenios y brindar } \\
\text { soluciones integrales desde la }\end{array}$ \\
\hline
\end{tabular}


Dom. Cien., ISSN: 2477-8818

Vol. 3, núm. 3, mon., agosto, 2017, pp. 811-828

Propuesta del Plan de Manejo Ambiental de las Lagunas de Mojanda: Caricocha, Huarmicocha y Yanacocha del cantón Pedro Moncayo

\begin{tabular}{|c|c|c|c|}
\hline sociedad) & & realización de proyecto. & academia. \\
\hline $\begin{array}{c}\text { Estudiantes de la } \\
\text { Carrera de Ingeniería } \\
\text { Ambiental }\end{array}$ & $\begin{array}{c}\text { No existe un Propuesta de } \\
\text { Plan de Manejo Ambiental en } \\
\text { el área de estudio. }\end{array}$ & $\begin{array}{l}\text { Elaborar la propuesta del } \\
\text { Plan de Manejo Ambiental } \\
\text { en el área de estudio. }\end{array}$ & $\begin{array}{l}\text { Proponer una Propuesta Plan } \\
\text { de Manejo Ambiental para la } \\
\text { conservación de la zona. }\end{array}$ \\
\hline Parroquia La Esperanza & $\begin{array}{l}\text { Pérdida de los ecosistemas } \\
\text { en el área de estudio. }\end{array}$ & $\begin{array}{c}\text { Brindar apoyo para la } \\
\text { conservación de los } \\
\text { ecosistemas en el área de } \\
\text { estudio. }\end{array}$ & $\begin{array}{l}\text { Convertir al área de estudio } \\
\text { en una zona turística, } \\
\text { ambientalmente sostenible. }\end{array}$ \\
\hline $\begin{array}{l}\text { Ministerio deAmbiente } \\
\text { del Ecuador (MAE) }\end{array}$ & $\begin{array}{l}\text { No recibir la documentación } \\
\text { reglamentaria para la } \\
\text { declaración de área } \\
\text { protegida. }\end{array}$ & $\begin{array}{c}\text { Recibir la documentación } \\
\text { para declararla como área } \\
\text { protegida. }\end{array}$ & Conservar ecosistemas. \\
\hline Ministerio de Turismo & $\begin{array}{l}\text { Bajo desarrollo turístico por } \\
\text { problemas de litigio. }\end{array}$ & $\begin{array}{c}\text { Solucionar los problemas } \\
\text { de litigio. }\end{array}$ & $\begin{array}{l}\text { Promover el turismo de la } \\
\text { zona. }\end{array}$ \\
\hline Turistas & $\begin{array}{l}\text { Acceso restringido por ser } \\
\text { propiedad privada. }\end{array}$ & $\begin{array}{l}\text { Exista libre acceso del } \\
\text { público a la zona. }\end{array}$ & $\begin{array}{l}\text { Contar con un área de } \\
\text { recreación natural. }\end{array}$ \\
\hline
\end{tabular}

Tabla 5. Matriz de involucrados

Elaborado por: Autores

Así mismo se obtuvo el árbol de problemas y objetivos del proyecto. 
Dom. Cien., ISSN: 2477-8818

Vol. 3, núm. 3, mon., agosto, 2017, pp. 811-828

Propuesta del Plan de Manejo Ambiental de las Lagunas de Mojanda: Caricocha, Huarmicocha y Yanacocha del cantón Pedro Moncayo

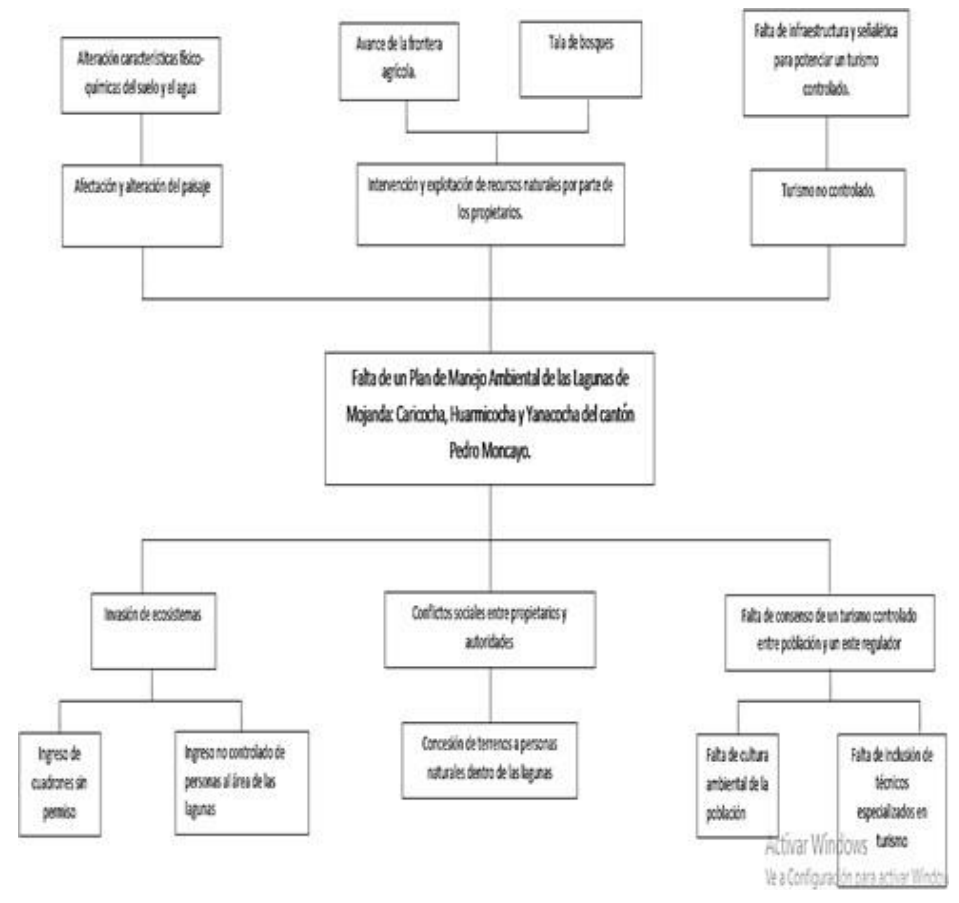

Figura 3. Árbol de problemas

Elaborado por: Autores

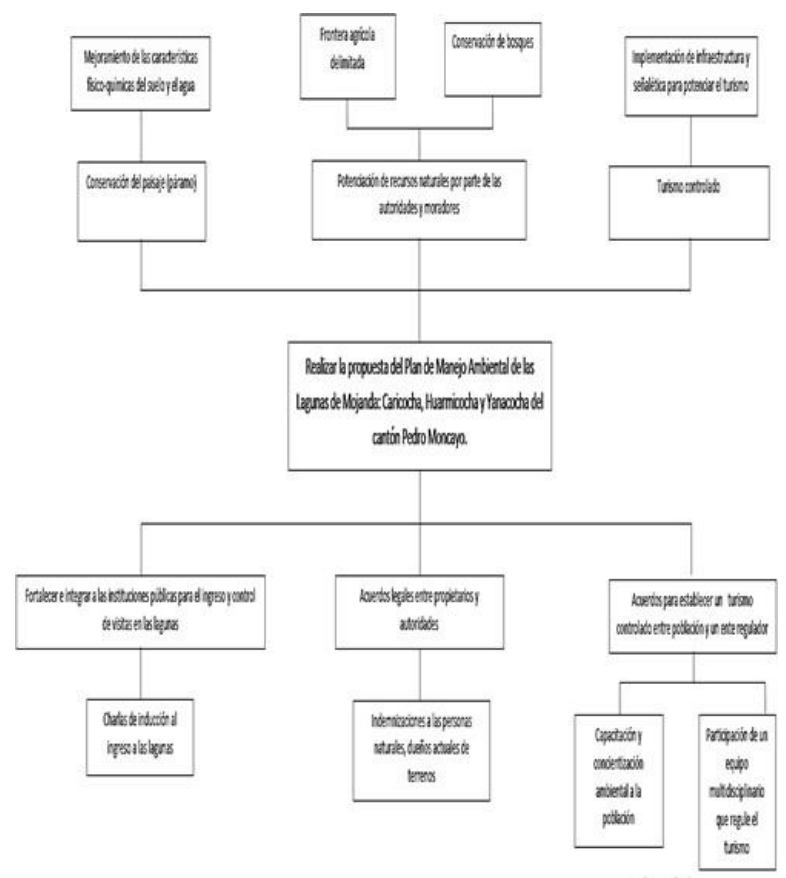

Figura 3. Árbol de problemas

Elaborado por: Autores 
Dom. Cien., ISSN: 2477-8818

Vol. 3, núm. 3, mon., agosto, 2017, pp. 811-828

Propuesta del Plan de Manejo Ambiental de las Lagunas de Mojanda: Caricocha, Huarmicocha y Yanacocha del cantón Pedro Moncayo

Al final de la aplicación de la metodología Marco lógico se obtuvo la Matriz de Marco Lógico:

\begin{tabular}{|c|c|c|c|}
\hline DESCRIPCIÓN & INDICADORES & FUENTE DE VERIFICACIÓN & SUPUESTOS \\
\hline FIN & \multirow[b]{2}{*}{$\begin{array}{c}\left(\mathrm{N}^{\circ} . \text { Declaratoria }\right. \\
\text { emitida/\# } \\
\text { Declaratoria } \\
\text { solicitada }){ }^{*} 100\end{array}$} & \multirow[b]{2}{*}{$\begin{array}{c}\text { Decreto Ejecutivo mediante el cual se } \\
\text { designa a las lagunas como área } \\
\text { protegida. }\end{array}$} & \multirow[b]{2}{*}{$\begin{array}{c}\text { MAE aprueba la } \\
\text { declaración de las } \\
\text { lagunas como área } \\
\text { protegida. }\end{array}$} \\
\hline $\begin{array}{l}\text { Declarar como área protegida a } \\
\text { las Lagunas de Mojanda: } \\
\text { Caricocha, Huarmicocha y } \\
\text { Yanacocha del cantón Pedro } \\
\text { Moncayo para contribuir al manejo } \\
\text { sustentable de las mismas. }\end{array}$ & & & \\
\hline $\begin{array}{l}\text { PROPÓSITO } \\
\text { Elaborar la propuesta del Plan de } \\
\text { Manejo Ambiental de las Lagunas } \\
\text { de Mojanda: Caricocha, } \\
\text { Huarmicocha y Yanacocha del } \\
\text { GAD Pedro Moncayo. }\end{array}$ & $\begin{array}{c}\left(\mathrm{N}^{\circ} \text {. programas }\right. \\
\text { ejecutados / \# } \mathrm{N}^{\circ} \text {. } \\
\text { programas } \\
\text { planificados) *100 }\end{array}$ & $\begin{array}{l}\text { Propuesta del Plan de manejo } \\
\text { Ambiental. }\end{array}$ & $\begin{array}{l}\text { No haya } \\
\text { organización y } \\
\text { participación de } \\
\text { los grupos } \\
\text { involucrados en el } \\
\text { proyecto. }\end{array}$ \\
\hline $\begin{array}{l}\text { C1: Plan de Manejo Ambiental } \\
\text { planificado }\end{array}$ & $\begin{array}{c}\left(\mathrm{N}^{\circ} . \text { programas de }\right. \\
\text { fases del plan de } \\
\text { manejo elaborado / } \\
\mathrm{N}^{\circ} \text {. programas } \\
\text { fases del plan de } \\
\text { manejo planificado) } \\
{ }^{*} 100\end{array}$ & $\begin{array}{c}\text { Informe de ejecución y revisión de las } \\
\text { fases de planificación del plan de manejo } \\
\text { ambiental integrado. }\end{array}$ & $\begin{array}{l}\text {-No se respete el } \\
\text { plan establecido. } \\
\text { - No haya acceso } \\
\text { y apertura a la } \\
\text { información } \\
\text { necesaria. }\end{array}$ \\
\hline $\begin{array}{l}\text { C2: Diagnóstico de la situación } \\
\text { actual de manejo del área y su } \\
\text { problemática elaborado }\end{array}$ & $\begin{array}{l}\left(\mathrm{N}^{\circ} \text {. programasde }\right. \\
\text { etapas del } \\
\text { diagnóstico } \\
\text { situacional } \\
\text { realizado } / \mathrm{N}^{\circ} \text {. } \\
\text { programasde } \\
\text { etapas del } \\
\text { diagnóstico } \\
\text { situacional } \\
\text { planificados) }{ }^{*} 100\end{array}$ & $\begin{array}{l}\text { Informe del diagnóstico situacional, } \\
\text { registro fotográfico, registros de } \\
\text { asistencia, listas de chequeo }\end{array}$ & $\begin{array}{c}\text {-No haya acceso y } \\
\text { apertura a la } \\
\text { información } \\
\text { necesaria }\end{array}$ \\
\hline $\begin{array}{l}\text { C3: Documento base del Plan de } \\
\text { Manejo y sus diferentes } \\
\text { programas ejecutados }\end{array}$ & $\begin{array}{l}\mathrm{N}^{\circ} \text {. programasde } \\
\text { programas de } \\
\text { manejo } \\
\text { elaborados } / \mathrm{N}^{\circ} \text {. } \\
\text { programasde } \\
\text { programas de } \\
\text { manejo } \\
\text { planificados) }{ }^{*} 100\end{array}$ & $\begin{array}{l}\text { Informe del plan de manejo ambiental, } \\
\text { registro fotográfico, registros de } \\
\text { asistencia, listas de chequeo }\end{array}$ & $\begin{array}{l}\text {-No exista el } \\
\text { tiempo suficiente } \\
\text { para presentar el } \\
\text { plan }\end{array}$ \\
\hline
\end{tabular}


Dom. Cien., ISSN: 2477-8818

Vol. 3, núm. 3, mon., agosto, 2017, pp. 811-828

Propuesta del Plan de Manejo Ambiental de las Lagunas de Mojanda: Caricocha, Huarmicocha y Yanacocha del cantón Pedro Moncayo

\begin{tabular}{|c|c|c|c|c|}
\hline \multicolumn{2}{|c|}{$\begin{array}{l}\text { C4: Plan de manejo ambiental } \\
\text { socializado al GAD de Pedro } \\
\text { Moncayo }\end{array}$} & \multirow{3}{*}{$\begin{array}{c}\left(\mathrm{N}^{\circ} \text {. programasde }\right. \\
\text { socializaciones } \\
\text { realizadas } / \mathrm{N}^{\circ} \text { de } \\
\text { socializaciones } \\
\text { planificadas) }{ }^{*} 100 \\
\\
\begin{array}{c}(\text { Ha del Área } \\
\text { delimitada/ Ha del } \\
\text { Área Total) }{ }^{*} 100\end{array}\end{array}$} & \multirow{3}{*}{$\begin{array}{l}\text { Informes de socializaciones del plan de } \\
\text { manejo, registro fotográfico, videos, y } \\
\text { registros de asistencia } \\
\text { Mapas digitales }\end{array}$} & \multirow{3}{*}{\begin{tabular}{|c|c|}
-Falta de \\
asistencia e \\
interés de los \\
actores \\
involucrados \\
\\
$\begin{array}{c}\text { Falta de presión } \\
\text { en mapas por } \\
\text { escalas grandes }\end{array}$ \\
\end{tabular}} \\
\hline \multicolumn{2}{|c|}{ ACTIVIDADES } & & & \\
\hline \multirow[b]{2}{*}{ Diagnóstico } & $\begin{array}{l}\text { Delimitación del } \\
\text { área de estudio }\end{array}$ & & & \\
\hline & \begin{tabular}{lr|}
\multicolumn{2}{l}{ Determinación } \\
de & situación \\
actual & del \\
complejo & de \\
lagunas &
\end{tabular} & $\begin{array}{c}\text { ( } \mathrm{N}^{\circ} \text {. programasde } \\
\text { aspectos } \\
\text { ambientales } \\
\text { positivos } / \mathrm{N}^{\circ} \text {. } \\
\text { programasaspecto } \\
\mathrm{s} \text { ambientales } \\
\text { negativos) }{ }^{*} 100\end{array}$ & Documento Línea Base & $\begin{array}{l}\text { Falta de apoyo } \\
\text { comunitario. } \\
\text { Errores de } \\
\text { medición. Falta de } \\
\text { equipos de } \\
\text { medición. Falta de } \\
\text { apertura a la } \\
\text { información } \\
\text { necesaria }\end{array}$ \\
\hline \multirow{2}{*}{$\begin{array}{l}\text { Elaboración de } \\
\text { la ficha técnica } \\
\text { para la } \\
\text { presentación de } \\
\text { proyectos }\end{array}$} & $\begin{array}{l}\text { Reunión para la } \\
\text { revisión del } \\
\text { documento }\end{array}$ & $\begin{array}{l}\left(\mathrm{N}^{\circ} . \text { programasde }\right. \\
\text { reuniones } \\
\text { realizadas } / \mathrm{N}^{\circ} . \\
\text { programasde } \\
\text { reuniones } \\
\text { programadas })^{*} 100\end{array}$ & Registro de asistencia & Falta de quórum \\
\hline & $\begin{array}{l}\text { Correcciones } \\
\text { del documento }\end{array}$ & $\begin{array}{l}\left(\mathrm{N}^{\circ} \text {. programas }\right. \\
\text { ítems corregidos/ } \\
\mathrm{N}^{\circ} \text {. programas } \\
\text { ítems por } \\
\text { corregir })^{\star} 100\end{array}$ & Control de cambios & $\begin{array}{c}\text { No exista } \\
\text { unanimidad para la } \\
\text { corrección, }\end{array}$ \\
\hline $\begin{array}{l}\text { Presentación de } \\
\text { la ficha Técnica } \\
\text { ante la } \\
\text { coordinación de } \\
\text { vinculación con } \\
\text { la sociedad }\end{array}$ & $\begin{array}{l}\text { Envío de la } \\
\text { ficha técnica al } \\
\text { departamento } \\
\text { encargado }\end{array}$ & $\begin{array}{l}\text { (Ficha técnica } \\
\text { aprobada/ficha } \\
\text { enviada) }{ }^{\star} 100\end{array}$ & Ficha técnica aprobada & $\begin{array}{l}\text { Que no exista } \\
\text { conformidad de la } \\
\text { ficha técnica. }\end{array}$ \\
\hline \multirow{2}{*}{$\begin{array}{l}\text { Elaboración del } \\
\text { documento del } \\
\text { Plan Integral de } \\
\text { Manejo } \\
\text { Ambiental }\end{array}$} & $\begin{array}{l}\text { Elaboración del } \\
\text { programa de } \\
\text { administración, } \\
\text { control y } \\
\text { vigilancia }\end{array}$ & $\begin{array}{l}\left(\mathrm{N}^{\circ} \text { de mejoras de }\right. \\
\text { la administración } \\
\text { ejecutadas } / \mathrm{N}^{\circ} \text { de } \\
\text { mejoras de la } \\
\text { administración } \\
\text { detectadas }){ }^{*} 100\end{array}$ & Memoria técnica & $\begin{array}{l}\text { No exista el tiempo } \\
\text { suficiente para } \\
\text { presentar el plan }\end{array}$ \\
\hline & $\begin{array}{|ll|}\text { Elaboración } & \text { de } \\
\text { Programa de } & \text { Planificación }\end{array}$ & $\begin{array}{c}\left(\mathrm{N}^{\circ} \text { de asistentes / }\right. \\
\mathrm{N}^{\circ} \text { total de } \\
\text { población }){ }^{*} 100\end{array}$ & Documento plan de capacitación & $\begin{array}{l}\text { Metodología y } \\
\text { elementos a } \\
\text { transmitir no } \\
\text { acordes al nivel }\end{array}$ \\
\hline
\end{tabular}


Dom. Cien., ISSN: 2477-8818

Vol. 3, núm. 3, mon., agosto, 2017, pp. 811-828

Propuesta del Plan de Manejo Ambiental de las Lagunas de Mojanda: Caricocha, Huarmicocha y Yanacocha del cantón Pedro Moncayo

\begin{tabular}{|c|c|c|c|}
\hline participativa & & & sociocultural \\
\hline $\begin{array}{l}\text { Elaboración } \\
\text { deprograma de } \\
\text { desarrollo } \\
\text { comunitario y } \\
\text { educación } \\
\text { ambiental }\end{array}$ & $\begin{array}{l}\left(\mathrm{N}^{\circ} \text { de conflictos }\right. \\
\text { sociales } /\left(\mathrm{N}^{\circ} \text { de }\right. \\
\text { relaciones } \\
\text { comunitarias }) \\
{ }^{*} 100\left(\mathrm{~N}^{\circ} \text { riesgos }\right. \\
\text { ocupacionales } \\
\text { controlados / } \mathrm{N}^{\circ} \\
\text { riesgos } \\
\text { encontrados }){ }^{*} 100\end{array}$ & $\begin{array}{l}\text { Documento plan de capacitación. } \\
\text { Memoria técnica }\end{array}$ & $\begin{array}{l}\text { Falta de apoyo } \\
\text { comunitario, Falta } \\
\text { de apertura a la } \\
\text { información } \\
\text { necesaria. No } \\
\text { exista el tiempo } \\
\text { suficiente para } \\
\text { presentar el plan }\end{array}$ \\
\hline $\begin{array}{l}\text { Elaboraciónpro } \\
\text { grama de } \\
\text { turismo } \quad \text { y } \\
\text { recreación }\end{array}$ & $\begin{array}{l}\text { ( } \mathrm{N}^{\circ} \text { de Actividades } \\
\text { turísticas } \\
\text { implementadas / } \\
\left(\mathrm{N}^{\circ} \text { de Actividades }\right. \\
\text { identificadas }){ }^{*} 100\end{array}$ & Memoria técnica & $\begin{array}{l}\text { No exista el tiempo } \\
\text { suficiente para } \\
\text { presentar el plan. } \\
\text { Falta de apoyo } \\
\text { comunitario. Poca } \\
\text { rentabilidad de } \\
\text { reaprovechamient } \\
\text { o. Poca } \\
\text { rentabilidad de } \\
\text { reaprovechamient } \\
\text { o }\end{array}$ \\
\hline $\begin{array}{l}\text { Elaboración de } \\
\text { programa de } \\
\text { investigación, } \\
\text { manejo de } \\
\text { recursos y } \\
\text { monitoreo }\end{array}$ & $\begin{array}{l}\left(\mathrm{N}^{\circ} \text {. de monitoreos }\right. \\
\text { realizados } /\left(\mathrm{N}^{\circ} \text { de }\right. \\
\text { monitoreos } \\
\text { planificadas }){ }^{* 100}\end{array}$ & Registros, asistencias. Memoria técnica & $\begin{array}{l}\text { Falta de apoyo } \\
\text { comunitario. } \\
\text { Falta de apertura a } \\
\text { la información } \\
\text { necesaria }\end{array}$ \\
\hline $\begin{array}{l}\text { Elaboración de } \\
\text { programa de } \\
\text { sostenibilidad } \\
\text { financiera }\end{array}$ & $\begin{array}{l}\left(\mathrm{N}^{\circ} . \text { Mecanismos }\right. \\
\text { financieros } \\
\text { proyectados }) /\left(\mathrm{N}^{\circ} \text {. }\right. \\
\text { mecanismos } \\
\text { financieros } \\
\text { identificados }) \times 100\end{array}$ & Proyección de gastos & $\begin{array}{c}\text { Falta de apertura a } \\
\text { la información } \\
\text { necesaria }\end{array}$ \\
\hline $\begin{array}{l}\text { Charla } \\
\text { informativa del } \\
\text { plan integrado } \\
\text { de manejo } \\
\text { ambiental de }\end{array}$ & $\begin{array}{l}\left(\mathrm{N}^{\circ} \text { de personas }\right. \\
\text { que entendieron y } \\
\text { apoyan el plan / } \\
\left(\mathrm{N}^{\circ} \text { de personas }\right. \\
\text { totales }){ }^{*} 100\end{array}$ & Registros, asistencias & $\begin{array}{l}\text { Falta de apoyo } \\
\text { comunitario }\end{array}$ \\
\hline
\end{tabular}

Tabla 6. Matriz de marco lógico Elaborado por: Ing. Carolina Artieda 
Dom. Cien., ISSN: 2477-8818

Vol. 3, núm. 3, mon., agosto, 2017, pp. 811-828

Propuesta del Plan de Manejo Ambiental de las Lagunas de Mojanda: Caricocha, Huarmicocha y Yanacocha del cantón Pedro Moncayo

Se realizó el análisis FODA del proyecto y se definió lo siguiente:

\begin{tabular}{|c|c|}
\hline \multicolumn{2}{|r|}{ FORTALEZAS } \\
\hline Sociales & $\begin{array}{l}\text { - Disposición de la población civil a participar en la vigilancia para la conservación } \\
\text { y protección de las Lagunas de Mojanda. } \\
\text { Delegados del GAD de Pedro Moncayo comprometidos con la labor de } \\
\text { protección y denuncia ciudadana. } \\
\text { Interés de la población en los atractivos y conocimiento sobre los recursos } \\
\text { naturales existentes en las lagunas. }\end{array}$ \\
\hline Económicas & $\begin{array}{l}\text { - } \text { El área cuenta con un complejo turístico totalmente equipado administrado por } \\
\text { el GAD Municipal del Cantón Pedro Moncayo. } \\
\text { - } \quad \text { al área de influencia propuesta para la declaración de Área de Conservación } \\
\text { tiene vías de acceso, infraestructura turística y conexión eléctrica. } \\
\text { La belleza escénica de las Lagunas de Mojanda proporciona beneficios } \\
\text { económicos a los habitantes que habitan alrededor ya que incrementa la } \\
\text { plusvalía de los terrenos. } \\
\text { Los negocios privados como hoteles y restaurantes también se ven } \\
\text { beneficiados económicamente por las Lagunas ya que les aporta un paisaje } \\
\text { escénico de magnífica belleza, el cual disfrutan turistas y la población en } \\
\text { general. }\end{array}$ \\
\hline Ambientales & 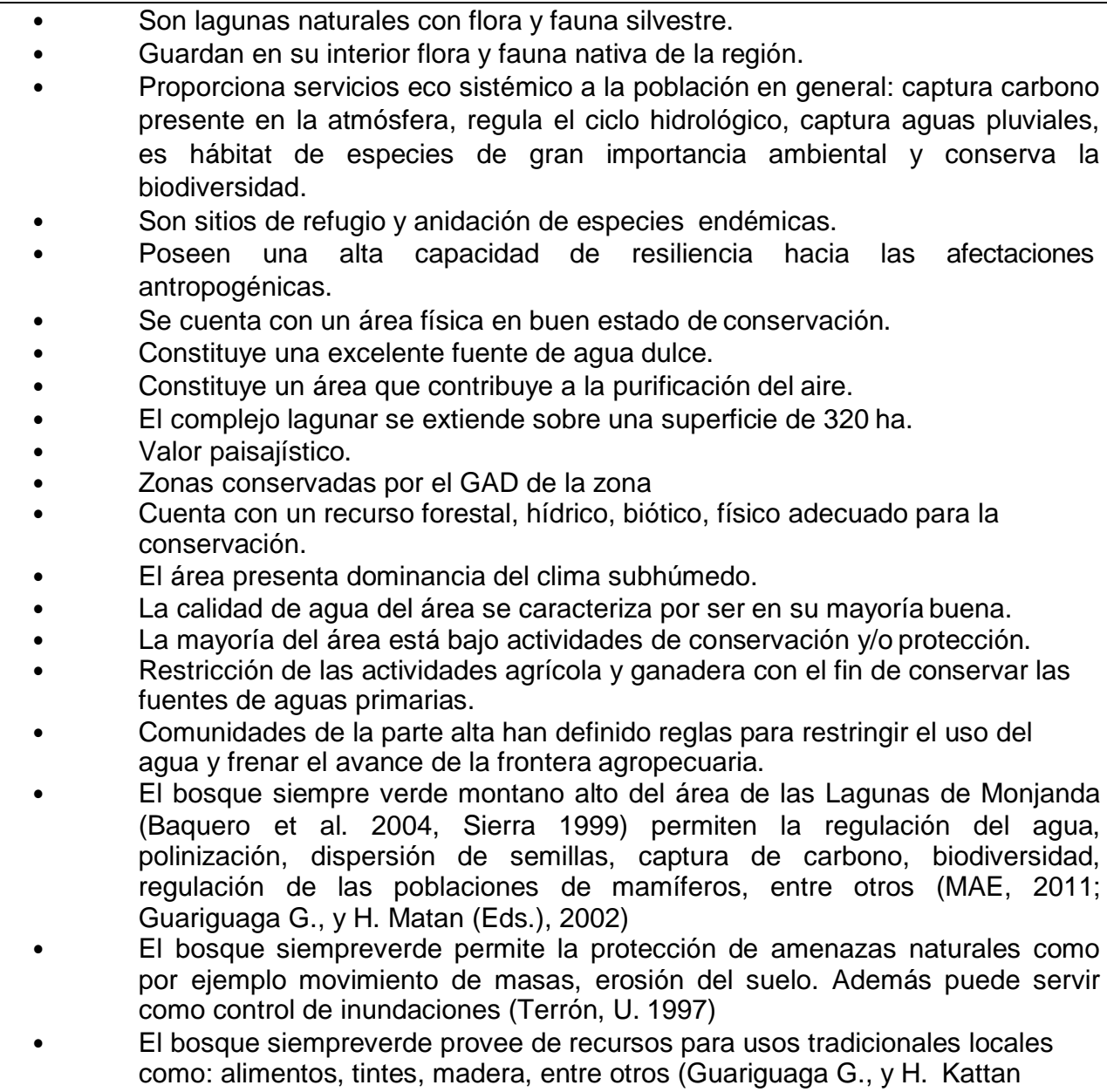 \\
\hline
\end{tabular}


Dom. Cien., ISSN: 2477-8818

Vol. 3, núm. 3, mon., agosto, 2017, pp. 811-828

Propuesta del Plan de Manejo Ambiental de las Lagunas de Mojanda: Caricocha, Huarmicocha y Yanacocha del cantón Pedro Moncayo

\begin{tabular}{|c|c|}
\hline & $\begin{array}{l}\text { (Eds.), 2002)); Ríos, et al. 2007) } \\
\text { - Su sistema hídrico asegura la capacidad de captación, absorción y regulación } \\
\text { de agua de la capa vegetal, para asegurar la disponibilidad de agua en la } \\
\text { prestación de servicios ecológicos. (Falconí. 2002) }\end{array}$ \\
\hline Legislativas & $\begin{array}{l}\text { Existencia de leyes, normas y reglamentos a nivel nacional que protegen y } \\
\text { garantizan la conservación y protección de las Lagunas de Mojanda. }\end{array}$ \\
\hline \multicolumn{2}{|r|}{ DEBILIDADES } \\
\hline Sociales & $\begin{array}{l}\text { - Falta de cultura ambiental. } \\
\text { - } \quad \text { Falta de seguimiento de las acciones a favor de la conservación de las } \\
\text { Lagunas de Mojanda. } \\
\text { - } \quad \text { Afectaciones civiles hacia la laguna, basura, desechos sólidos contaminantes } \\
\text { - } \quad \text { Dalta de conciencia ambiental. } \\
\text { Desobediencia por parte de los visitantes a los reglamentos y advertencias del } \\
\text { gobierno y del GAD. } \\
\text { Escaso conocimiento por parte de los pobladores de los bienes y servicios eco } \\
\text { sistémicos. }\end{array}$ \\
\hline Económicas & $\begin{array}{l}\text { - } \\
\text { - } \quad \text { hontro de las Lagunas de Mojanda. } \\
\text { Falta de recursos para llevar a cabo monitoreos periódicos dentro de las lagunas } \\
\text { de Mojanda. } \\
\text { - } \quad \text { Falta de incentivos para promover la conservación y actividades } \\
\text { No existe una planificación a largo plazo. }\end{array}$ \\
\hline Ambientales & $\begin{array}{l}\text { - } \\
\text { - } \quad \text { Incendios } \\
\text { Deforestación } \\
\text { - } \quad \text { Contaminación del agua } \\
\text { - } \quad \text { Nauna vulnerable a los ataques civiles y falta de regulación } \\
\text { - } \quad \text { establecimiento de corredores de conservación. } \\
\text { Existen } 16.202 \text { ha }(58,3 \%) \text { de cobertura vegetal; en las cuales el paisaje natural } \\
\text { ha sido modificado, conformando una zona consolidada de intervención, en la } \\
\text { cual se diferencian áreas cubiertas por cultivos, pastizales y bosques plantados } \\
\text { con especies exóticas(eucalipto y pino) } \\
\text { Adquisición de terrenos adjudicados por el IERAC, dentro del área a conservar. } \\
\text { Existe escasa información sobre la importancia del bosque siempreverde del } \\
\text { - área de conservación. } \\
\text { Las características geomorfológicas de la región hace a los sistemas hídricos } \\
\text { vulnerables a contaminación por efectos de actividades antrópicas. } \\
\text { Ausencia de un Plan de Manejo de las Lagunas de Mojanda. }\end{array}$ \\
\hline Legislativas & $\begin{array}{l}\text { - Falta de acuerdos para aplicar la legislación vigente } \\
\text { - } \quad \text { Existencia de afectaciones ambientales permanentes en las Lagunas sin } \\
\text { resolución o sanción del gobierno } \\
\text { - } \quad \text { Fscases de procesos de participación publica } \\
\text { particulares que generen beneficios a la sociedad como programas de educación } \\
\text { ambiental, recorridos turísticos, avistamientos de fauna, navegación en las } \\
\text { lagunas. }\end{array}$ \\
\hline \multicolumn{2}{|r|}{ OPORTUNIDADES } \\
\hline Sociales & $\begin{array}{l}\text { - Hay disponibilidad por parte de la sociedad de participar en la conservación y } \\
\text { conocimiento de las Lagunas de Mojanda. } \\
\text { Disponibilidad del GAD de Pedro Moncayo de brindar su apoyo para solucionar }\end{array}$ \\
\hline
\end{tabular}


Dom. Cien., ISSN: 2477-8818

Vol. 3, núm. 3, mon., agosto, 2017, pp. 811-828

Propuesta del Plan de Manejo Ambiental de las Lagunas de Mojanda: Caricocha, Huarmicocha y Yanacocha del cantón Pedro Moncayo

\begin{tabular}{|c|c|c|}
\hline & & $\begin{array}{l}\text { los problemas de contaminación del área. } \\
\text { Apoyo interinstitucional para usar, preservar o conservar el páramo } \\
\text { Capacitación a la población para un adecuado manejo de los recursos naturales } \\
\text { y conservación de los páramos. } \\
\text { Las instituciones locales apoyadas por la sociedad civil están interesadas en } \\
\text { buscar alternativas para conservación de los bosques siempreverdes. }\end{array}$ \\
\hline Económicas & • & $\begin{array}{l}\text { Generación de empleo al facilitar el uso de las Lagunas para llevar a cabo } \\
\text { actividades recreativas y ecoturismo } \\
\text { Demanda de turismo ecológico }\end{array}$ \\
\hline Ambientales & $\bullet$ & $\begin{array}{l}\text { Disponibilidad de una línea base de investigación que proporciona información } \\
\text { importante sobre las condiciones de la flora, fauna, aspecto físico y paisajístico } \\
\text { de las Lagunas } \\
\text { Disponibilidad de personal calificado para llevar a cabo monitoreos permanentes } \\
\text { dentro y fuera de las Lagunas. } \\
\text { Ejecución de acciones de protección y conservación del área para mantener las } \\
\text { funciones ecológica }\end{array}$ \\
\hline Legislativas & $\bullet$ & $\begin{array}{l}\text { Elaboración de un Estudio de alternativas de manejo para la creación del área } \\
\text { ecológica de conservación de las Lagunas de Mojanda. } \\
\text { Apoyo del GAD de Pedro Moncayo para llevar a cabo el proceso de área } \\
\text { protegida. } \\
\text { Formar parte del Sistema Nacional de Áreas Protegidas SNAP. }\end{array}$ \\
\hline
\end{tabular}

\section{Tabla 7. Matriz FODA}

Elaborado por: Autores

El contenido de cada uno de los programas del plan de manejo ambiental, se

especifica a continuación:

1. Programa de administración, control y vigilancia: busca proporcionar pautas para mejorar la administración del Área "Lagunas de Mojanda", donde se involucre a la comunidad e instituciones relacionadas con el proyecto, logrando así conservar la integridad física y ecológica del área.

2. Programa de planificación participativa: busca que la comunidad de las "Lagunas de Mojanda", participe en forma activa y decisoria en la elaboración del plan de desarrollo de su comunidad.

3. Programa de desarrollo comunitario y educación ambiental: busca mejorar la calidad de vida e incorporar la participación activa de las comunidades aledañas al Área de Conservación "Lagunas de Mojanda".

4. Programa de Turismo y Recreación: busca potenciar el desarrollo turístico en el Área de Conservación “Lagunas de Mojanda”, de manera sistemática y planificada. 
5. Programa de investigación, manejo de recursos y monitoreo: buscaconservar y monitorear las actividades que se realizan en las "Lagunas de Mojanda".

6. Programa de Sostenibilidad Financiera: busca generar recursos económicos para la sostenibilidad financiera en el Área de Conservación "Lagunas de Mojanda", de manera planificada.

\section{Discusión.}

El trabajo con la metodología de Marco Lógico permitió plantear dos grandes áreas: la primera referente a los fundamentos y diagnósticos que sustentan el plan de manejo ambiental donde se utilizan herramientas tales como el "árbol de problemas" y el "árbol de objetivos" y la segunda referente a la conceptualización del proyecto y se basa en una matriz con la información básica del plan denominada "Matriz de Marco Lógico".

En la Propuesta de Plan de Manejo Ambiental de las "Lagunas de Mojanda" se realizaron 6 programas que permiten establecer acciones para prevenir, mitigar, controlar, compensar y corregir los posibles efectos o impactos ambientales negativos causados por las actividades desarrolladas en el área de laslas Lagunas de Mojanda: Caricocha, Huarmicocha y Yanacocha, con el fin de que de que a futuro esta zona se la declare como área protegida, en cada programa se analizó el objetivo del mismo, se definió objetivos específicos, líneas de acción, actividades, indicadores, medio de verificación y plazo para cumplimientos.

\section{Conclusiones.}

Los programas de la Propuesta de Plan de Manejo Ambiental de las Lagunas de Mojanda: Caricocha, Huarmicocha y Yanacocha, permiten establecer acciones para prevenir, mitigar, 
controlar, compensar y corregir los posibles efectos o impactos ambientales negativos

causados por las actividades desarrolladas en el área.

\section{Recomendaciones.}

Que los indicadores de las actividades de cada programa de la Propuesta del Plan de Manejo de lasLagunas de Mojanda: Caricocha, Huarmicocha y Yanacocha, sean evaluados para determinar el nivel cumplimiento.

\section{Bibliografía.}

[1].Ministerio del Ambiente. (2015). Metodología de Gestión del Destino de Áreas Naturales Protegidas. Obtenido de http://www.rainforestalliance.org/sites/default/files/publication/pdf/metodologi\%CC\%81a\%20en\%20baja.pdf

[2].Ambiente, M. d. (2015). Lineamientos para la elaboración de planes de sostenibilidad financiera para el Patrimonio de Áreas Naturales del Estado. Quito - Ecuador.

[3].Ministerio del Ambiente. (06 de Julio de 2016). Ministerio del Ambiente. Obtenido de http://www.ambiente.gob.ec/

[4].Organización de las Naciones Unidas para la Alimentación y la Agricultura. (07 de Julio de 2016). Organización de las Naciones Unidas para la Alimentación y la Agricultura. Obtenido de http://www.fao.org/home/es/ 